\title{
Optimising Operation Process for Computer Integrated Prostatectomy
}

\author{
Q. Mei $^{1}$, S. J. Harris ${ }^{1}$, R. D. Hibberd ${ }^{1}$, J. E. A. Wickham ${ }^{2}$, and B.L.Davies ${ }^{1}$ \\ ${ }^{1}$ Mechatronics in Medicine Laboratory, Imperial College, London SW7 2BX, England \\ 2 Department of Surgery, Guys Hospital, London SE1, England
}

\begin{abstract}
A method to optimise the whole process of operation/vaporising the prostate tissue using a prostatectomy robot to relieve urethra blockage is presented. The cavity that closely satisfies the surgeon defined model can be created by robot in half of the time that done manually. The desired vaporising region can even be reshaped during the operation, resulting in new optimised vaporising sequence that excludes the region already operated. The method also excluds vulnerable regions from operation for safety. All models maintained benefit for pre/intra/post-operation evaluation. The method exhibits a promising future for the application of robotic prostatectomy and robotic surgery on soft tissue in general.
\end{abstract}

\section{Introduction}

The PROBOT (PROSTATE-CTOMY ROBOT), a computer integrated prostatectomy system, has been developed in the MIM Lab, Imperial College to aid in the resection of prostate tissue. [Fig. 1]. Aspects of the design that have been considered are the graphical user interface, safety of the hardware and software, the use of real-time ultrasound imaging, 3D modelling, sterilisation of the system etc [1-3,9]. The PROBOT has already been tried on 18 patients with satisfactory result

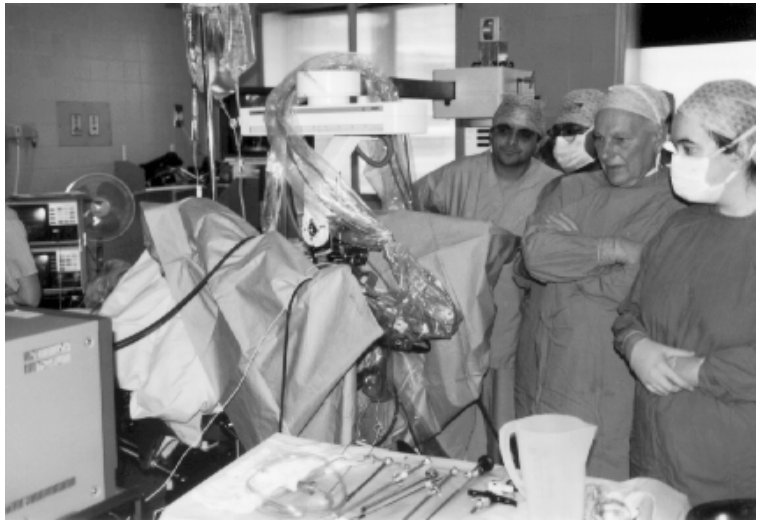

Fig. 1- the PROBOT is in operation (The surgeon is monitoring the screen) except that the overall operation time is 
approximately 1.5 times slower than its manual counterpart [9].

This paper mainly focuses on the optimisation of the vaporising process. Improved with new optimising software of the operation/vaporising process, the PROBOT can be expected to finish the operation within half of the time that done manually.

Prostate enlargement is a common disease among men over certain age and quite often this results in a blockage of urethra for which an operation called TURP (TransUrethral Resection of Prostate) is required to create a cavity inside the prostate and restore the normal passage of urine. A typicalmanual operation of such takes about 40 minutes or less to finish and takes away about 10 grams of prostate tissue [8].

By nature, the prostate can be regarded as a soft object that is fixed in position inside the men's body. The region around the urethra between penis and prostate is very flexible which allows the vaporising instruments to move transurethrally in different angles. When being operated upon, the inside of the prostate may deform locally, but the prostate as a whole generally maintains both its position and shape. A rigid model can thus be generated and processed based on transurethral ultrasound image slices, which makes the robotised operation feasible.

An essential part of the PROBOT is a specially designed motorised frame that is capable of creating a cone shaped cavity at desired positions [Fig. 2]. Mounted on the frame with an ultrasound probe, automatic ultrasound imaging can be carried out. Otherwise mounted with vaporising instrument, cystoscope and video camera, the system can perform prostatectomy operation, or non-bleeding vaporising procedure with clear on-line video monitoring.

The strategy for optimising the operation/vaporising process is based on matching the surgeon defined regions on recorded US images with corresponding pre-arranged, maximum mechanically feasible, overlapping cones, to generate the vaporising sequence with least number of vaporising actions (or vaps).
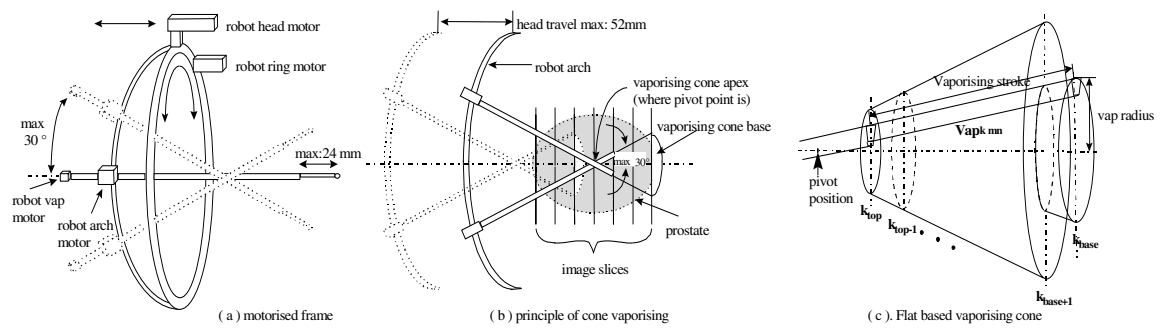

Fig. 2 - Model of the motorised frame and principle of (extended) cone-shape vaporising

\section{Registration}

Registration is normally concerned with relating the reference systems associated with different modalities to the rigid or elastic geometric transforms [4-6]. However, the registration of PROBOT is very straightforward. At the beginning of the surgery, the bladder neck and verumontanum (a clearly visible landmark that should never be 
resected) are first registered to the PROBOT via direct viewing from the cystoscope. Then, automatic transurethral ultrasound imaging is performed between the two end positions of the prostate. US (ultrasound) image slices, perpendicular to the urethra or central axis, are recorded at $5 \mathrm{~mm}$ intervals in the computer by means of a frame grabber integrated into the system. Subsequently, any geometric element on these image slices is directly transferred into the co-ordinate system of the motorised frame. The physical distortion from the 'soft' feature of prostate tissue caused by the imaging process has a negligible effect, as this distortion will be the same for the vaporising instrument because they share the same working mechanism.

\section{Surgeon Defined Model}

Having acquired computer based US images, the surgeon can now mark on each what region or area should be removed. A 3D surgeon defined model is thus obtained:

$$
\mathbf{M o d e l}_{\text {def }}=\left\{\mathbf{A r e a}_{\text {def } i}\right\} \text { and } \mathbf{A r e a}_{\text {def } i}=\left\{\text { dist }_{\text {def } i j}\right\} \quad \mid(\mathbf{i}=0,1, \cdots, \mathbf{I} ; \mathbf{j}=0,1, \cdots, 359)
$$

Where Area $_{\text {def i }}$ is a surgeon defined area on ith image slice; $\left\{\right.$ dist $_{\text {def }}$ ij $\}$ is the boundary for Area def $i$ and dist $_{\text {def } i j}$ is the distance from the centre (urethra) to the boundary, at individual radial angle $\mathbf{j}$ on ith image; $\mathbf{I}$ is the largest index number of an image.

The anterior surfaces of prostate are covered by abundant loose fat that includes the preprostatic venous plexus [7], which is a vulnerable region susceptible to heavy bleeding during prostatectomy operation. As shown in Fig. 3, this region is located in the upper $120^{\circ}$ part of the prostate, and slightly away from the urethra or where the

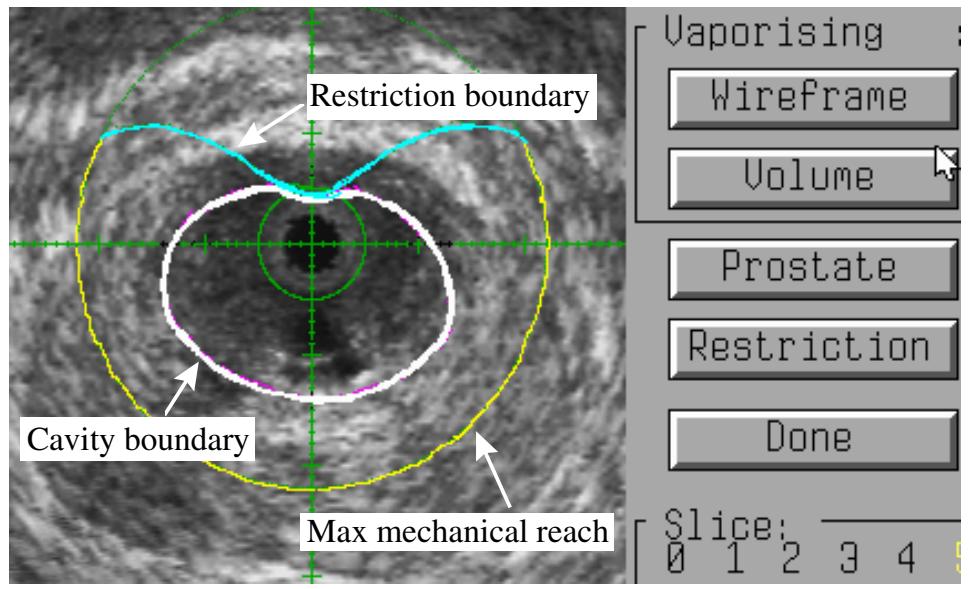

Fig. 3- Restriction boundary for safe cavity model definition 
sheath of the motorised frame is anchored. A restriction boundary is given there, so that this vulnerable region will be excluded from the operation. The restriction boundary can be pre-set or edited to satisfaction for each slice. Together with the boundary of maximum mechanical reach on other parts, a region is formed where surgeon can define the boundary of cavity model.

\section{Mechanically Feasible Model}

One way to create the cavity of surgeon defined model is to cover it with a number of maximum "mechanically feasible" overlapping cones. By careful planning, the relationship between the distance of the verumontanum and bladder_neck, and the least number of cones, is worked out as that shown in Table 1. These cones are positioned from the verumontanum towards the bladder_neck at $5 \mathrm{~mm}$ intervals. The opening radius of the cone at the verumontanum is controlled by press_depth (a parameter from the user interface) for the purpose of reducing the risk of hitting the verumontanum. An example is shown in Fig. 4.

Table 1 - Relationship between the distance between bladder neck and verumontanum and total number of cones

\begin{tabular}{|c|c|c|c|c|c|c|}
\hline Dist (veru - bladder) & $<=25 \mathrm{~mm}$ & $<=33 \mathrm{~mm}$ & $<=38 \mathrm{~mm}$ & $<=43 \mathrm{~mm}$ & $<=48 \mathrm{~mm}$ & $<=52 \mathrm{~mm}$ \\
\hline Number of slices & $<=6$ & $=6 / 7$ & $=7 / 8$ & $=8 / 9$ & $=9 / 10$ & $=10 / 11$ \\
\hline Number of cones & 1 & 2 & 3 & 4 & 5 & 6 \\
\hline
\end{tabular}

(The distance between verumontanum slice and its neighbouring slice can be $1 / 2 / 3 / 4 / 5 \mathrm{~mm}$; otherwise always $5 \mathrm{~mm}$ )

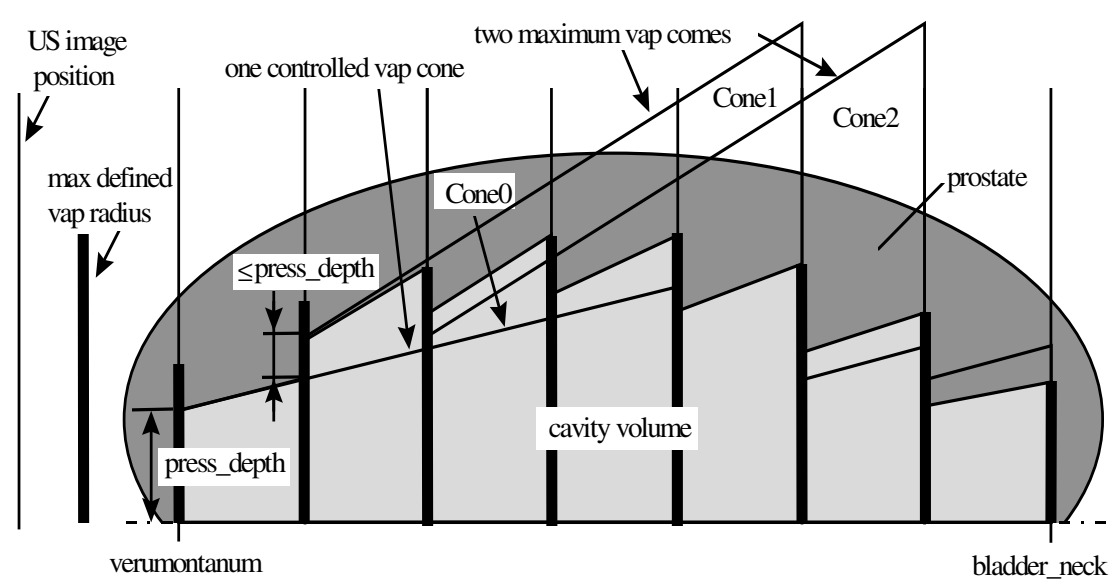

Fig. 4 - Section diagram of using pre-arranged cones to maximumly cover the desired vaporising volume 
Given a surgical case, the corresponding mechanically feasible vaporising model can be obtained:

$$
\operatorname{Model}_{\text {fea }}=\bigcup_{\mathrm{k}=0}^{\mathrm{k}=\mathrm{K}} \text { Cone }_{\mathrm{fea} \mathrm{k}}
$$

$$
\text { Cone }_{\text {fea } k}=\left\{\operatorname{vap}_{\mathrm{kmn}}\right\} \mid\left(\mathbf{m}=1,2, \cdots, \mathbf{M}_{\mathrm{k}} ; \mathbf{n}=0,1, \cdots, \mathbf{N}_{\mathrm{mk}}\right)
$$

Where $\mathbf{K}$ is the largest possible index number of a cone. Each cone consists of a series of vaporising actions which starts from the inside to outside, and first in a clockwise then anti-clockwise way (to avoid entangling wires). $\mathbf{M}_{\mathbf{k}}$ is the number of vaporised rings of cone $\mathbf{k} ; \mathbf{N}_{\mathbf{m k}}$ is the largest number of a vap for ring $\mathbf{m}$ of cone $\mathbf{k}$. Both $\mathbf{M}_{\mathbf{k}}$ and $\mathbf{N}_{\mathbf{m k}}$ are controlled by vap_depth, which controls how deep each vap will vaporise the tissue, and vap_width, which controls the distance between each neighbouring vaps. These also come from the user interface.

The above model can also be expressed as

$$
\text { Model }_{\text {fea }}=\left\{\text { Area }_{\text {fea i }}\right\} \quad \mid(\mathbf{i}=0,1, \cdots, \mathbf{I})
$$

Where Area fea is the intersection of pre-arranged vaporising cones and slice $\mathbf{i}$, or:

$$
\text { Area }_{\text {fea }}=\bigcup \text { area }_{\mathbf{i k m n}} \mid\left(\mathbf{k}=0,1, \cdots, \mathbf{K} ; \mathbf{m}=1,2, \cdots, \mathbf{M}_{\mathbf{k}} ; \mathbf{n}=0,1, \cdots, \mathbf{N}_{\mathrm{mk}}\right)
$$

Where $\operatorname{area}_{\mathbf{i k m}}$ is the intersection of $\operatorname{vap}_{\mathbf{k} \mathbf{m}}$ and slice $\mathbf{i}$.

\section{The Final Vaporising Model}

Obviously, part of the pre-arranged mechanically feasible model may operate outside the surgeon defined model. Appropriate adjustments should be made so that there should be no vaps to operate outside the Model $_{\text {def }}$.

In other words, the final vaporising model should be, ideally, the intersection of the surgeon defined model and the mechanically feasible model, which can be expressed as follows:

$$
\begin{aligned}
& \text { Model }_{\text {final }}=\left\{\text { Area' }_{i}\right\} \quad \mid(\mathbf{i}=0,1, \cdots, \mathbf{I}) \\
& \text { Area' }_{i}=\bigcup \text { area' }_{\mathbf{i k m n}}
\end{aligned}
$$

Where area' $_{\mathbf{i k m n}}=\operatorname{vap}_{\mathbf{k ~ m n}} \cap$ Area $_{\text {def } i} \mid\left(\mathbf{k}=0,1, \cdots, \mathbf{K} ; \mathbf{m}=1,2, \cdots, \mathbf{M}_{\mathbf{k}} ; \mathbf{n}=0,1, \cdots, \mathbf{N}_{\mathrm{mk}}\right)$

$$
\text { or } \text { Model }_{\text {final }}=\left\{\text { vap' }_{\mathrm{kmn}}\right\}
$$

where each vap' ${ }_{\mathbf{k} \text { mn }}$ operates within Model $_{\text {def }}$ 


\section{Optimising the Generation of Vaporising Sequence}

The aim of optimising the vaporising sequence is to generate from $\mathbf{M o d e l}_{\text {final }}$, a vaporising sequence that will be finished in the shortest time. From the design of PROBOT's motorised frame, the control parameters for $\boldsymbol{l}$ th vaporising action ( $\left.\mathbf{v a p}_{l}\right)$ are:

1. The distance to the pivot from the last position on the central axis to control the head travel motor $\left(\mathbf{h}_{l}\right)$

2. The eccentric angle of the vap off the central axis to control the $\operatorname{arch}$ motor $\left(\mathbf{a}_{l}\right)$

3. The angle of the vap off $0^{\circ}$ home position on slice to control the ring motor $\left(\mathbf{r}_{l}\right)$

4. The vaporising stroke to control the vap motor $\left(\mathbf{d}_{l}\right)$

Let the vaporising sequence be: $\left[\operatorname{vap}_{l}\left(\mathbf{h}_{l}, \mathbf{a}_{l}, \mathbf{r}_{l}, \mathbf{d}_{l}\right)\right], \mathbf{1} \leq \boldsymbol{l} \leq \mathbf{L}$ (L is the total number of vaps) and $\mathbf{h}_{\mathbf{0}}, \mathbf{a}_{\mathbf{0}}, \mathbf{r}_{\mathbf{0}}, \mathbf{d}_{\mathbf{0}}$ be the origin of all motors. The total time $\mathbf{T}$ spent on executing this vaporising sequence can be calculated by the sum of the time for each vaps $\left(\mathbf{t}_{l}\right)$ :

$$
\mathbf{T}=\sum \mathbf{t}_{l} \quad \mathbf{1} \leq l \leq \mathbf{L}
$$

$\mathbf{t}_{l}$ can be calculated by the sum of the $\mathbf{t}_{\mathrm{h} l}, \mathbf{t}_{\mathrm{a} l}, \mathbf{t}_{\mathrm{rl}}$, and $\mathbf{t}_{\mathrm{d} l}$, where $\mathbf{t}_{\mathrm{h} l}$ is the time spent for the head travel to move the sheath to the given pivot position $\mathbf{h}_{l}$ from $\mathbf{h}_{l-1} ; \mathbf{t}_{\mathbf{a l}}:$ arch rotation from $\mathbf{a}_{l-1}$ to $\mathbf{a}_{l} ; \mathbf{t}_{\mathbf{r} l}$ : ring rotation from $\mathbf{r}_{l-1}$ to $\mathbf{r}_{l}$; and $\mathbf{t}_{\mathrm{d} l}$ : vaporising motor for a stroke(forward and backward). The time spent on communication can be neglected. So we have:

$$
\mathbf{t}_{l}=\mathbf{t}_{\mathrm{h} l}+\mathbf{t}_{\mathrm{a} l}+\mathbf{t}_{\mathrm{r} l}+\mathbf{t}_{\mathrm{d} l}
$$

The optimisation objective is to minimise $\mathbf{T}$. This could be decomposed into the following five sub-objectives: i.e., to minimise $\mathbf{L}$; minimise $\mathbf{t}_{\mathrm{h} l}, \mathbf{t}_{\mathrm{al}}, \mathbf{t}_{\mathrm{r} l} ;$ and $\mathbf{t}_{\mathrm{d} l}$.

Since these five parameters are not independent of each other, a reasonable principle is to give higher priority to the more time consuming parameters. Based on experience with PROBOT, priority has been set in the following sequence:

1. Minimise $\mathbf{L}$

2. Minimise $\mathbf{t}_{\mathrm{h} l},, \mathbf{t}_{\mathrm{a} l}, \mathbf{t}_{\mathbf{r} l}$

3. Minimise $\mathbf{t}_{\mathbf{d} l}$

4. Minimise $\mathbf{t}_{\mathrm{h} l}, \mathbf{t}_{\mathrm{a} l}, \mathbf{t}_{\mathrm{r} l}$

To minimise $\mathbf{L}$ (the total number of vaps), it is necessary to maximise the amount for each vap or to use a full stroke where possible. It is also necessary to avoid repetitive vaps, which means it has to be remembered where all the previous vaps have reached, so that the subsequent vaps can be generated more effectively.

When vaporising a positioned single cone, $\mathbf{t}_{\mathrm{h} l}$ will be zero, $\mathbf{t}_{\mathrm{a} l}$ and $\mathbf{t}_{\mathrm{r} l}$ will be a small constant (decided by vap_depth and vap_width). $\mathbf{t}_{\mathbf{d} l}$ is decided by the vaporising stroke.

Based on the analysis above, an optimisation algorithm called max-stroke vaporising by cones has been developed. It always finds, among the cones left, the one that has the maximum stroke. 


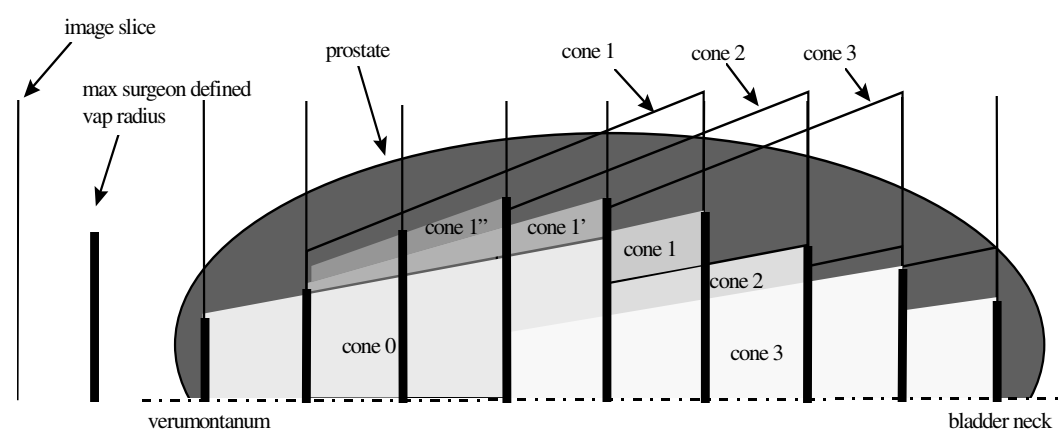

Fig. 5- Principle of max-stoke vaporising by cones

The principle of the algorithm is shown in Fig. $\mathbf{5}$ and the 3D shape of the cavity is shown in Fig. 6. Without optimisation, to finish the cavity of model ${ }_{\text {def }}$, the total time spent will be the summation of time spent on 4 individual cones (cone 0 to 3), or, the time is proportional to the summation of the section areas of 4 individual cones. With optimising, the total time is only proportional to the union of these 4 section areas.

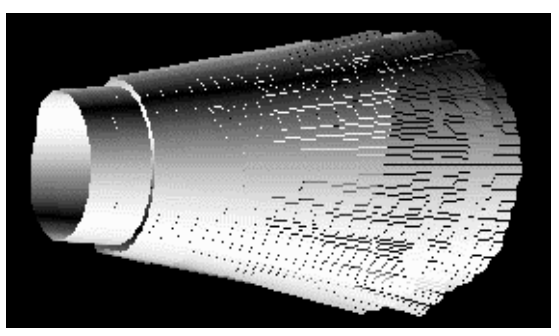

Fig. 6 - An Example of 3D shape of the cavity created by PROBOT

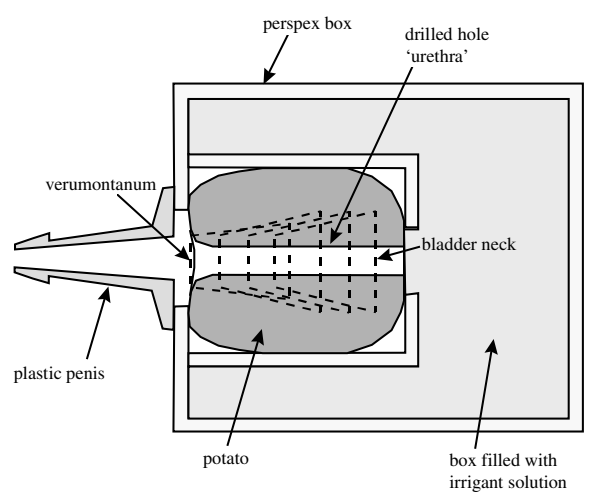

Fig. 7- Potato test settings

Moreover, a new mechanism of two way vaporising (both forward and backward vaporising) is introduced, by which a further $1 / 3$ of operation time can be saved comparing to one way vaporising (backward only).

\section{Results of Experiments}

The system with optimisation has gone through 25 potato tests [9], a traditional practice before live patient trial [Fig. 7]. Repeatability is within $1.5 \mathrm{~mm}$ on the maximum diameter of the cavity and $5 \mathrm{~mm}$ of cone spacing is good [Fig. 9]. 
It can be seen, from Table 2, the difference between the maximum diameter defined and maximum diameter obtained is within 0.75 $\mathrm{mm}$ with standard deviation being within $0.51 \mathrm{~mm}$.

Table 3 and Fig. 8 show the time (minutes) spent on vaporising procedures. It can be seen that with two way cutting, to create a cavity of $10 \mathrm{cc} / \mathrm{gram}$, the vaporising procedure can be finished in approximately 20 minutes, which is the half of the time that done manually. For the largest prostate (about $50 \mathrm{~mm}$ long with 11 slices) that PROBOT is able to operate, the two way vaporising procedure can be finished in less than half an hour.

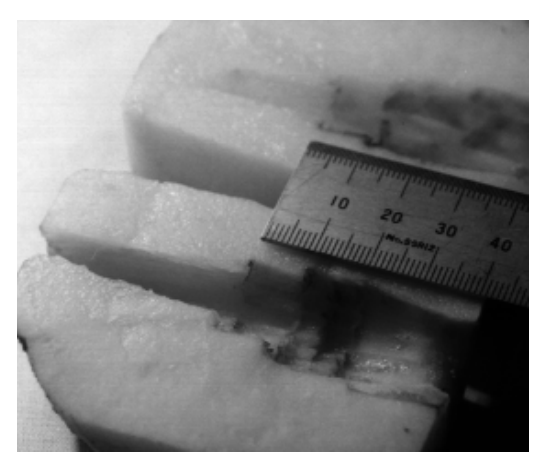

Fig. 9 - An example of the section shape of the potato after the "operation"

\section{Fig. 8 - Relationship between cavity volume and vaporising time with vap_width setting at 4.4 $\mathrm{mm}$ and vap_depth $2.5 \mathrm{~mm}$}
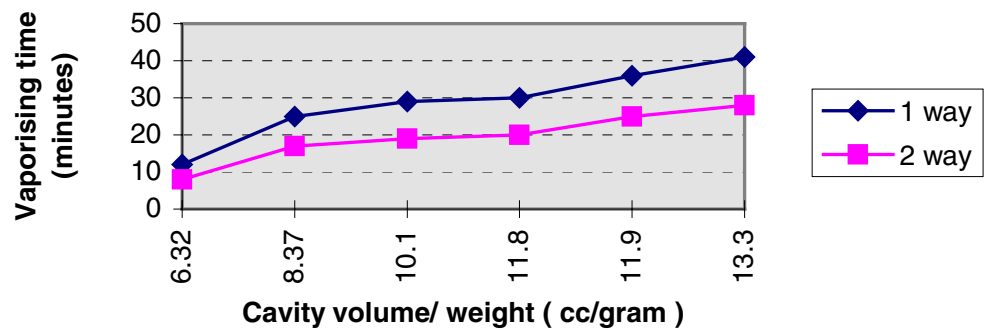

Table 2 - Experiment data 1

\begin{tabular}{|c|c|c|cc|}
\hline $\begin{array}{c}\text { length } \\
\mathbf{m m}\end{array}$ & $\begin{array}{c}\text { num } \\
\text { Cases }\end{array}$ & $\begin{array}{c}\text { Max } \\
\text { Def } \mathbf{D}\end{array}$ & $\begin{array}{l}\text { max vap } \\
\text { standard }\end{array}$ & $\begin{array}{c}\text { D mean \& } \\
\text { deviation }\end{array}$ \\
\hline 25 & 3 & $23 \mathrm{~mm}$ & 22.75 & - \\
\hline 30 & 2 & $21 \mathrm{~mm}$ & 20.25 & 0.35 \\
\hline 35 & 11 & $24 \mathrm{~mm}$ & 23.67 & 0.51 \\
\hline 40 & 2 & $22 \mathrm{~mm}$ & 21.25 & 0.35 \\
\hline 45 & 5 & $22 \mathrm{~mm}$ & 21.4 & 0.42 \\
\hline 50 & 2 & $22 \mathrm{~mm}$ & 21.25 & 0.35 \\
\hline
\end{tabular}

Vap_width: 4mm; vap_depth $1.7 \mathrm{~mm}$
Table 3 - Experiment data 2

\begin{tabular}{|c|c|c|c|c|c|c|}
\hline Test & slices & press & vaps & volume & 1 way (min) & 2way (min) \\
\hline 1 & 6 & 3.5 & 49 & 6.32 & 12 & 8 \\
2 & 7 & 3.5 & 105 & 8.37 & 25 & 17 \\
3 & 8 & 3 & 122 & 10.06 & 29 & 19 \\
4 & 9 & 2.5 & 128 & 11.78 & 30 & 20 \\
5 & 10 & 2 & 154 & 11.87 & 36 & 25 \\
6 & 11 & 2 & 173 & 13.33 & 41 & 28 \\
\hline
\end{tabular}

Vap width: $4.4 \mathrm{~mm}$; vap depth: $2.5 \mathrm{~mm}$ which are acceptable by urologists. 


\section{Regenerating the Optimised Vaporising Sequence}

While vaporising is in process, it is likely to occur that the surgeon defined cavity model needs to be reshaped because of, say, hitting blood vessels or being too close to the capsule of the prostate. Then the software needs to re-generate the optimised vaporising sequence that should efficiently exclude the region that has already been vaporised rather than start vaporising from the scratch.

The surgeon re-defined model can be expressed again as:

$$
\begin{array}{ll}
\operatorname{Model}_{\text {re-def }}=\left\{\text { Area }_{\text {re-def }}\right\} & \mid(\mathbf{i}=0,1, \cdots, \mathbf{I}) \\
\text { Area }_{\text {re-defi }}=\left\{\text { dist }_{\text {re-def } i j}\right\} & \mid(\mathbf{j}=0,1, \cdots, 359)
\end{array}
$$

The model for the cavity region that has already been created can also be expressed as:

$$
\begin{aligned}
& \mathbf{M o d e l}_{\text {vaped }}=\left\{\text { Area }_{\text {vaped } \mathrm{i}}\right\} \quad \mid(\mathbf{i}=0,1, \cdots, \mathbf{I}) \\
& \text { Area }_{\text {vaped } i}=\left\{\text { dist }_{\text {vaped ij }}\right\} \quad \mid(\mathbf{j}=0,1, \cdots, 359)
\end{aligned}
$$

So the required vaporising model can be expressed as:

$$
\begin{aligned}
& \operatorname{Model}_{\text {def }}=\left\{\text { Area }_{\text {def } \mathrm{i}}\right\}=\left\{\text { Area }_{\text {redefi }}-\text { Area }_{\text {vaped }}\right\} \quad \mid(\mathbf{i}=0,1, \cdots, \mathbf{I}) \\
& \text { or } \mathbf{A r e a}_{\text {def i }}=\left\{\text { dist }_{\text {re-def } i j}-\text { dist }_{\text {vaped ij }}\right\} \quad \mid(\mathbf{j}=0,1, \cdots, 359)
\end{aligned}
$$

The generation of optimised vaporising sequence for this model is the same as that described in section 6 .

\section{Conclusions}

This paper describes different stages involved in optimising the operation/vaporising process in a computer integrated prostatectomy system. The pre-arranged maximum mechanically feasible overlapping cones ensure that the surgeon defined model is completely covered so that any unnecessary under-vaporising can be avoided. Generating the optimised vaporising sequence with max-stroke vaporising by cones ensures the sequence will be generated in the least number of vaporising actions. With two way vaporising, this sequence can be finished in half of the time that done manually. Re-generating the optimised vaporising sequence, to exclude the region already vaporised, makes the system more practical. Vulnerable regions are excluded from the operation. This adds to the system safety functionality. As the whole vaporising sequence is generated before the start of actual vaporising, the graphical simulation of the vaporising process can be fully realised $[3,9]$. While the vaporising procedure being finished automatically, surgeon can sit aside and just monitor, releasing him from otherwise a very demanding task [Fig. 1]. 
In short, after the above optimisation method is implemented, the prostatectomy operation carried out by PROBOT can be finished faster, safer, nicer and with better pre/intra/post-operation evaluations.

\section{References}

1. Davies, B. L., Hibberd, R.D., Ng, W.S., Timoney, A., Wickham, J.E.A., "Mechanical Constraints - The Answer to Safe Robotic Surgery?"' J.de Innovation et Technoogie en Biologie et Medecine, France, Vol. 13 No. 4. pp425-436, Sept. 1992.

2. Harris, S.J., Mei, Q., Arambula-Cosio, F., Hibberd, R.D., Nathan, MS, Wickham, JEA, Davies, B.L., “A Robotic Procedure for Transurethral Resection of the Prostate". Second Annual International Symposium on Medical Robotics and Computer Assisted Surgery, John Wiley \& Sons, Inc., Baltimore, USA, November 1995. pp264-271.

3. Mei, Q., Harris, S.J., Arambula-Cosio, F., Hibberd, R.D., Nathan, MS, Wickham, JEA, Davies, B.L., "PROBOT - A Computer Integrated Prostatectomy Robot System", Procedings of Fourth International Conference on Visualization in Biomedical Computing, Hamburg, Germany. Sept. 22-25, 1996

4. LAVALLÉE, S, et al., Image-Guided Operating Robot: A Clinical Application in Stereotactic Neurosurgery, p343-352, COMPUTER INTEGRATED SURGERY Technology and Clinical Applications, The MIT Press, 1996.

5. Kall, B. A. Computer-Assisted Surgical Planning and Robotics in Stereotactic Neurosurgery, p353-362, COMPUTER INTEGRATED SURGERY Technology and Clinical Applications, The MIT Press, 1996.

6. Taylor, R. H., et al., An Image-Directed Robotic System for Precise Orthopaedic Surgery, p379-398, COMPUTER INTEGRATED SURGERY Technology and Clinical Applications, The MIT Press, 1996.

7. Fornage, Bruno D. Ultrasound of the prostate, Chichester : Wiley Press, 1988.

8. Nathan, M.S., Wickham, J.E.A., "TVP: a cheaper and effective alternative to TURP", Minimum Invasive Therapy \& Allied Technology, 1996, :5: pp292 - 296.\#

9. Mei, Q., "PROBOT - A Computer Integrated Prostatectomy System", PhD thesis, Imperial College, May 1998. 